\title{
Demystifying online personas of Vietnamese young adults on Facebook: A Q-methodology approach
}

\author{
Duy Dang-Pham
}

School of Business IT and Logistics

RMIT University

duy.dang@rmit.edu.au

Siddhi Pittayachawan

School of Business IT and Logistics

RMIT University

siddhi.pittayachawan@rmit.edu.au

\author{
Mathews Nkhoma \\ Department of Business IT and Logistics \\ RMIT International University \\ Vietnam \\ mathews.nkhoma@rmit.edu.vn
}

\section{Abstract}

As online social networking sites (SNS) are becoming a part of many people's daily life, they have become a topic of interest that attracts the attention of both academics and practitioners. The users' behaviours on those sites are complex and have important implications. Individuals can use a wide range of SNS's functionalities, for example, to socialise and promote their online identities or find friends of common interests. Reckless online behaviours on SNS have been shown to result in breaches of privacy and other risks such as cyber-bullying, cyber-stalking, and job loss. As a result, there are many concerns over these problems that prevent SNS from reaching their full potential. Given the vast number of user's concerns, identified by prior studies, there is a need to explore a typology of users that classifies online personas. This typology would reveal more about the users' needs and help improve their experience on SNS. To achieve that goal, we collected data from fourteen Vietnamese young adults of different backgrounds who use Facebook, which was then analysed with $\mathrm{Q}$ factor analysis. The findings suggested three distinctive online personas that include the Outgoing Connector, the Reserved Trustor, and the Threats Perceiver. Implications for marketing tactics and design of better users' experiences on social networking sites were discussed, and the proposed typology could serve as a starting point for future research to further explore the different types of social media's users.

Keywords: Facebook; social networking site; Q methodology; onlineidentity; online privacy; information behaviour

\section{Introduction}

\subsection{Background}

Social networking sites (SNS) have been receiving tremendous attention not only from the end users but also practitioners and researchers who are interested in these virtual, multifaceted domains. By gaining more knowledge of the users' behaviours and preferences on SNS, better services and protection could be developed to cater their needs and enhance the users' online experiences. Furthermore, research about social media uses also brings implications to domains such as online education and business (A. Carrillat et al. 2014; Boyd and Ellison 2007). With the large amount of research investigating users' concerns about the different aspects of SNS, recent literature reviews that summarised these findings were able to explain why people use social media (e.g. Nadkarni and Hofmann 2012). Nevertheless, there remain opportunities to bring out more value from the existing research by studying them as a whole, especially when they have been examined separately as an attempt to gain an in-depth 
understanding about their respective topics. As a consequence, we are attempting to construct a typology of users based on their concerns that have been identified by prior studies. More specifically, we anticipate elaborating the relations between the concerns about different aspects and hence explore the unique personalities of the SNS users. Potential findings derived from the typology would add insights to the current discussion by explaining why different types of users are using social media in their own ways.

There are few studies that investigated typologies of online citizens. For instance, Meyen et al. (2010) grouped Internet users based on their everyday life's activities and types of capital (i.e. cultural and social) with qualitative analysis. Brandtzaeg and Heim (2011) used cluster analysis to classify users based on the intensity of their participation on SNS (i.e. high or low) and objectives of participation (i.e. informational or recreational). Their study collected data from 5,233 users on four popular SNS in Norway, and found five types of users that fall into the four quadrants as determined by the two mentioned criteria. Most recently, Hassouneh and Brengman (2014) took a mixed methods approach to explore traits of social virtual world users with exploratory factor analysis.

Our research is different from the mentioned typological studies in that we focus on the concerns of the end users about Facebook as a popular SNS, rather than the broader theme of Internet behaviours or more specific activities such as a virtual social game. Furthermore, the anticipated typology of personalities will be developed based on multiple concerns in relation to the aspects identified by prior studies such as identities, information sharing, online privacy, and so on. This scope allows the extracted personalities to provide more complete portraits of groups of users, rather than focusing solely on their motivations to use SNSs or intensity.

\subsection{Research context}

Of our particular interest is the SNS Facebook which has been growing rapidly since its first appearance in 2004. To date, it has reached more than 890 million daily active users from around the world (Facebook 2015). This SNS offers a wide range of functionalities that allow the users to customise their personal pages and profiles, as well as communicate with the others by privatemessaging or posting contents (e.g. updates, news, websites, music and video) on their pages. Facebook also hosts community's pages and online games where users sharing common interests can participate in and socialise. According to the 2014 global survey by Pew Research Center (2014), 87 per cent of young-adult Internet users whose ages range from 18 to 29 years old use Facebook, and it was found to attract the highest number of online adults when compared to other SNS. As Facebook's high popularity attracts end users of varied demographics, we decided to limit our investigation to the personalities of young adults who are the dominant cohort of users.

Our research context further focuses on Vietnamese young adult users of Facebook, whose concerns and use of social media are influenced by their unique cultural values as a part of the larger Asian context. Similar to other societies, Facebook is also popular among Vietnamese social media users. According to the latest report by WeAreSocial (2015) about Internet and social media usage around the world in J anuary 2015, Vietnam had a 40 per cent grouth of social media users (accounted for 31 per cent of total population) since 2014 when there were 20 million active Facebook accounts (WeAreSocial 2014). 


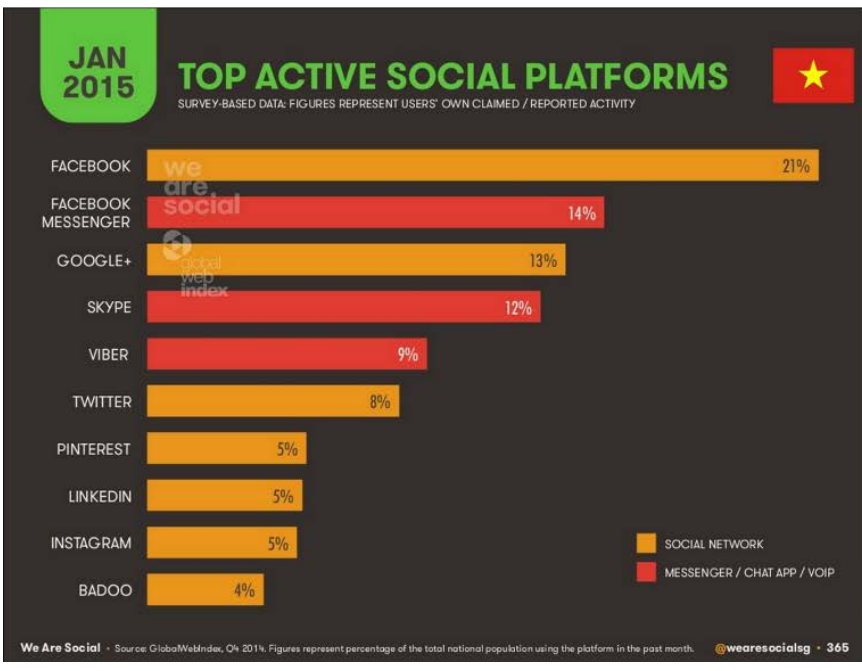

Social media sites, 2012-2014

\% of online adults whouse the following social media websites, by year

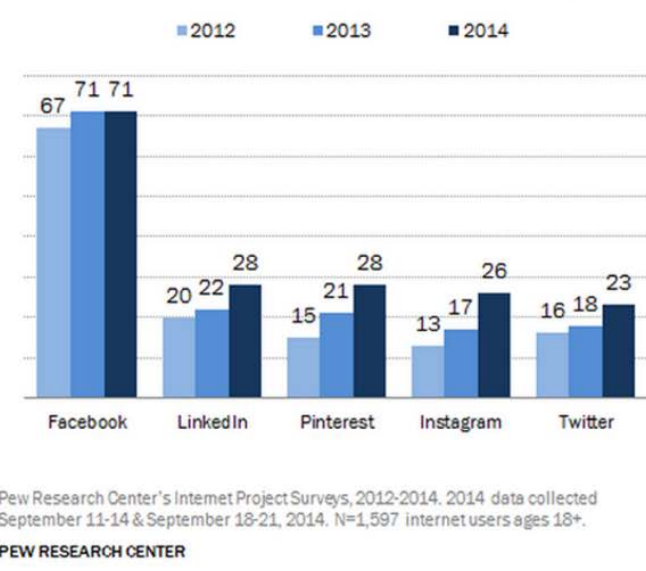

Figure 1: Facebook usage compared to other SNS by Vietnamese (left) and global users (right) - adapted from Pew Research Center (2014) and WeAreSocial (2015)

This present research is a part of a larger project which aims at capturing and comparing Vietnamese users' concerns on Facebook and Zing Me, a local SNS (www.zing.me) which has functionalities that resemble Facebook and was its main competitor in 2012 when both had 8.5 and 8.4 million users respectively (WeAreSocial 2012).

In this study, we focus on analysing and classifying the users' concerns on Facebook by performing Q factor analysis, so to explore the distinctive Facebook personas of Vietnamese young adult users. We start with consolidating concerns of SNS users by conducting a literature review in thenext section, to assist us in developing an instrument to collect data. The research methodology and analyses are then presented, followed by research findings and our discussion. Finally, we conclude our study with implications and limitations.

\section{Literature Review}

\subsection{Concerns about socialisation and functionalities}

One of the fundamental and critical aspects of most SNS is the user's disclosure of information which can be observed from their personal profile or communication with others (Kietzmann et al. 2011; Krasnova et al. 2010). Disclosure of information is fundamental as every user is required to submit their information (to some extent depending on the SNS) and to register for an SNS account, yet critical because the strategies for establishing and maintaining identities relate to the user's goals and concerns about other important aspects (Boyd and Ellison 2007). To begin with, one important implication of those strategies is for selfpromotion or self-branding (Kietzmann et al. 2011). These purposes, which are termed selfpresentation, are among the central desires that motivate users to disclose more information (Boyd 2007; Krasnova et al. 2010), especially to promote their attractive traits and receive social approvals (Mehdizadeh 2010). In particular, self-presentation can be achieved by disclosing many types of information, including those that are identifiable (e.g. real names, genders, and profile images) and more private such as hobbies, tastes of music and movies, relationship status, or sexual preferences (Gross and Acquisti 2005; Krasnova et al. 2010; Stutzman 2006).

In relation to identities, maintaining and building online relationships were regarded as the main benefits of using SNS and disclosing information (Krasnova et al. 2010). For example, the availability of accurate information such as names and pictures can allow other users to assess and validate the authenticity of one's account (Boyd and Ellison 2007). Likewise, Ellison et al. (2011) differentiate between making friends indiscriminately with those who share similar SNS contexts (e.g. playing the same games) and "social browsing", which refers to selectively finding more information about contacts that have similar offline backgrounds. In 
addition, users can also search for information about products and organisations, provided that online stores and organisations are leveraging social media to reach more potential customers (Kietzmann et al. 2011).

The next step after finding information about other people is establishing relationships with them. The studies of Lampe et al. (2006) and Joinson (2008) reveal that re-acquiring lost contacts or keeping in touch with close friends ranked highly in the list of purposes of using Facebook, followed by making new friends and having in-person encounter with people met online. Interestingly, Kietzmann et al. (2011) discussed connections with like-minded people and even potential dates result from conversations between individuals and groups on SNS. Furthermore, some users view SNS as a mean to acquire and accumulate social capital, especially by creating ties with new contacts that can provide them unique information (Krasnova et al. 2010). Similarly, users may expand their businesses and professional networks on SNS, provided that SNS have been recently adopted as a recruitment channel by organisations (A. Carrillat et al. 2014).

SNS offer a range of interactional functionalities that facilitate establishing identities and building relationships. Blogging is a type of interaction that conveys one's message to the audience, while online gaming and social groups or pages provide a common ground for finding people with similar interests (Ellison et al., 2011; J oinson, 2008; Kietzmann et al., 2011). Sharing content such as music, video, and photos is another way to make connections among individuals (J oinson 2008; Kietzmann et al. 2011). However, Kietzmann et al. (2011) discussed that whether sharing would result in a relationship between individuals depends on the shared objects fit with the SNS's functional objective (e.g. pictures for Flickr and careers for LinkedIn). Finally, Tan et al. (2012) found the SNS's perceived ease of use and usefulness motivates users to continue using it, which indicates the importance of the functions' usability.

\subsection{Concerns about threats of privacy}

Privacy threats have been a primary interest of scholarship concerning SNS since 2004 (Boyd and Ellison 2007). One of the earliest and widely cited articles about privacy on Facebook found the surveyed users reveal a generous amount of personal information while appearing unconcerned about their privacy (Gross and Acquisti 2005). Furthermore, Krasnova et al. (2010) discussed that the privacy risks on SNS include not only those mentioned in ecommerce's literature such as having information collected by third-party companies but also secret sharing, identity theft, and misuse of personal information (e.g. for phishing purposes). In addition, Krasnova et al. (2009) found the users were concerned about their personal data being collected and used by the SNS providers. Similarly, other researchers studying users' privacy on SNSs such as Stutzman et al. (2011) and Kietzmann et al. (2011) also discussed those threats in their studies. However, Debatin et al. (2009) suggested not every user would recognise these threats as they argued that organisations' violations of personal data such as data mining remain invisible to the average users.

Apart from the aforementioned privacy risks that involve losing control over personal data, there are several other threats that emerge from the online environment. Gross and Acquisti (2005), Debatin et al. (2009), and Krasnova et al. (2009) discussed social threats such as stalking (offline and online), bullying, and uncontrollable actions of other users such as inappropriate comments, harassment, or denigration. Moreover, Debatin et al. (2009) found rumours and gossips on Facebook play central roles by keeping the SNS interesting while causing social violations of privacy. Stories shared among the participants in these authors' focus group further illustrate privacy incidents in which insults were exchanged via posting of embarrassing comments and abusive tagging as well as modifying personal pictures. Nevertheless, Krasnova et al. (2009) found these social threats do not affect information disclosure, and they argued that such insignificant impact could be due to privacy controls perceived to be effective against social threats.

Moreover, there has been an emerging number of studies investigates the impact of SNS's contents on one's career. For example, the study by Karl et al. (2010) revealed that the surveyed participants would be inclined to post "problematic content" (e.g. self-photo drinking alcohol 
or being provocative) and private information on their profile such as religious beliefs and political affiliations. Similarly, Stoughton et al. (2013) studied posting of bad-mouthing behaviour and substance use in their research, and they shared the common view with Karl et al. (2010) that employers have been paying extra attention to inappropriate content for screening potential candidates and monitoring "off-the-clock" behaviour of their employees.

Similarly, users often weigh the benefits against the costs of disclosing information or making connections before initiating any social interaction, and online trust has been regarded as an important factor that mitigates the mentioned privacy threats as a major cost (Krasnova et al. 2010). Trust is multi-dimensional and contains several meanings which include the truster's assessment of the trustee's competence, predictability, benevolence, and integrity (Pittayachawan et al. 2008). Furthermore, Krasnova et al. (2010) argued that only the latter two would affect information disclosure as SNS do not hold specific topics that can be used to evaluate one's competence to converse on it. Nevertheless, discussing in-depth online trust is outside the scope of this research, and this present study will only focus on the user's trust in the SNSs' protective services and functions, as well as in their online friends who would not harm them.

In addition to trust is the user's perceived control over their information, which is reflected in how much they are aware of or using the privacy settings of the SNS (Krasnova et al. 2010). More specifically, these authors discussed that SNS providers need to have transparent policies about collecting and maintaining their users' information, as well as to give them clear tools to manage their privacy (e.g. limit access to personal profile, remove and report inappropriate contents). As a consequence, it is expected that multiple settings and effective usability of the SNS's privacy functions would be among the users' concerns.

\section{Research Methodology}

We conducted this study using Q-methodology which was developed by Stephenson (1953) to explore and classify SNS users into distinct groups or "online personas". This was achieved by using a combination of enquiries including Q sorts (i.e. card-sorting), semi-structured interviews, and observation. Each participant was requested to sort the cards from the "most concerned" to the "most unconcerned" issues on a scale, which ranges from +6 to -6 based on the distribution illustrated in Figure 2. Semi-structured interview was used before card-sorting to collected data about demographic and SNS experience, and after card-sorting to ask the participants why they sorted the cards in such a way, especially issues located at the extreme (i.e. +6 and -6 ) or middle positions (i.e. 0). The card-sorting data was then analysed with qmethod package in $\mathrm{R}$ (Zabala 2014). The interview data was used to aid interpretation of the findings.

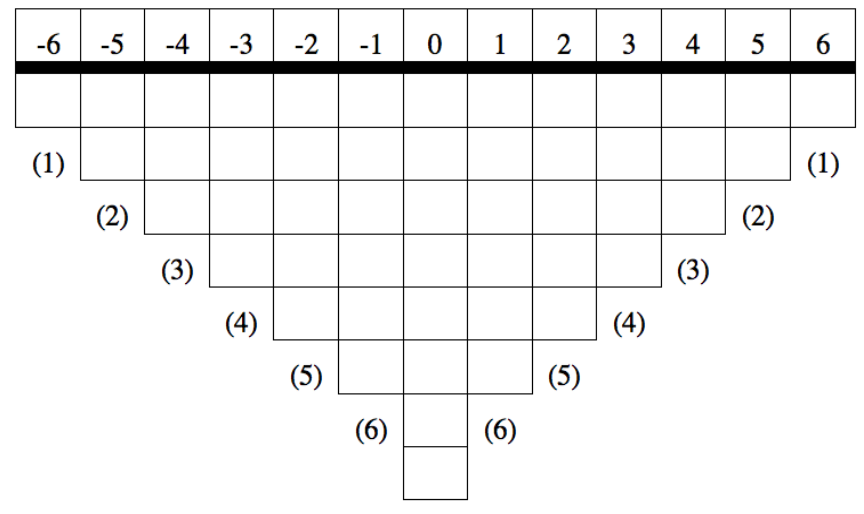

(8)

Figure 2: Q distribution 


\subsection{Development of the statements}

Since none of the prior studies used Q methodology, we had to adopt the idea from previous researches. According to Watts and Stenner (2012), a Q set should contain items with a good coverage related to the research question. We developed the statements for the sorting activity based on our literature review in the previous section. Specifically, we identified six major themes including Relationships, SNS functionalities, Interactions, Finding information, Privacy threats, and Identity. "Relationships" refers to the use of Facebook to connect with family and friends, or for purposes such as expanding professional network (Ellison et al. 2011; J oinson 2008; Kietzmann et al. 2011; Lampe et al. 2006). "SNS functionalities" includes items such as playing games, sharing music and video, as well as the usability and protective measures of Facebook (Ellison et al. 2011; J oinson 2008; Tan et al. 2012). "Interactions" lists out activities such as communications, sharing pictures, writing blog, and building online trust (Boyd 2007; Ellison et al. 2011; Krasnova et al. 2010). "Finding information" refers to the need to search for information about people, events, products, or to read online news (Kietzmann et al. 2011). "Privacy threats" covers threats such as insulting, online scams, identity theft, cyberstalking, and posting sensitive contents (Boyd and Ellison 2007; Debatin et al. 2009; Gross and Acquisti 2005; Stutzman et al. 2011). "Identity" describes the concerns over putting which types of information on the user's profile, as well as the need for self-presentation (Boyd 2007; Kietzmann et al. 2011; Mehdizadeh 2010). We composed the Q set's statements that reflect the content of the identified themes. The comprehensiveness of the statements, as well as their relation to the existing functionalities of Facebook, was carefully considered during the development process. The final list of 50 statements can be found in the Appendix.

\subsection{Profiles of the participants}

To recruit the participants, public advertisement that details the project's objectives and procedures has been posted on Facebook and online forums for one month. The participants were invited to attend the individual interview and Q-sort session at RMIT Vietnam University's campus in Ho Chi Minh City. Initial rejections to participate suggested that the card sorting procedure was perceived to be confusing as compared to the traditional survey method and traveling to the campus was inconvenient. Consequently, small gifts were used as incentives to encourage participation.

This research is a part of a larger project which aims at capturing and comparing users' concerns on Facebook and Zing Me, which is a popular Vietnamese SNS that resembles Facebook, and this paper only discusses the concerns of Facebook's users. As a result, one of the key selection criteria includes the participants must have used both Facebook and Zing Me for at least one year. Among the 24 participants who met our criteria and were set up appointments to participate in the project, only 14 attended to the actual sessions. The demographics of the 14 participants are displayed below, with the average age of 23.07 years old and an even ratio of genders.

\begin{tabular}{|c|c|c|c|c|c|c|c|}
\hline ID & Age & Gender & Occupation & ID & Age & Gender & Occupation \\
\hline 1 & 20 & Female & HE student & 2 & 25 & Male & IT consultant \\
\hline 5 & 21 & Female & HE student & 3 & 25 & Male & Pharmacist \\
\hline 7 & 21 & Female & HE student & 4 & 22 & Male & IT intern \\
\hline 9 & 23 & Female & Vet intern & 6 & 25 & Male & Marketer \\
\hline 10 & 21 & Female & HE student & 8 & 28 & Male & Marketer \\
\hline 11 & 21 & Female & HE student & 13 & 21 & Male & HE student \\
\hline 12 & 27 & Female & HR executive & 14 & 23 & Male & Accountant \\
\hline
\end{tabular}

Table 1: Demographics of participants

\subsection{Data analysis}

We used Principal Component Analysis with Spearman's correlation and Cluster rotation to identify three factors in $\mathrm{R}$ with the qmethod package. The total explained variance of the three 
factors is $49 \%$ (Table 2). One of the participants was not loaded on any factor (i.e. a confounded case), thereby being dropped from subsequent analysis. Factors 1 and 2 are clearly loaded by the majority of the interviewees. All factors are highly reliable $(>0.80)$.

\begin{tabular}{|l|r|r|r|}
\hline \multicolumn{1}{|c|}{ Participants } & Factor 1 & Factor 2 & Factor 3 \\
\hline 14F_XDAI & 0.792 & -0.141 & -0.053 \\
\hline 9F_DANOI & 0.741 & 0.168 & 0.087 \\
\hline 6F_DLONG & 0.591 & 0.299 & 0.08 \\
\hline 8F_KHANG & 0.584 & 0.146 & 0.114 \\
\hline 4F_LONGT & 0.487 & -0.185 & 0.317 \\
\hline 11F_NGA & 0.085 & 0.783 & -0.154 \\
\hline 5F_DIEM & -0.166 & 0.650 & 0.371 \\
\hline 7F_LINDA & 0.283 & 0.574 & -0.305 \\
\hline 13F_QHAI & -0.134 & 0.549 & 0.059 \\
\hline 10F_LIFE & 0.243 & 0.483 & 0.038 \\
\hline 12F_UYEN & 0.145 & -0.099 & 0.768 \\
\hline 1F_TRANG & -0.098 & 0.285 & 0.636 \\
\hline 2F_THINH & 0.453 & -0.178 & 0.555 \\
\hline \multicolumn{5}{|r|}{} & 5 & & \\
\hline $\mathrm{n}$ & 2.5 & 2.2 & 3 \\
\hline Eigenvalue & 19 & 17 & 13 \\
\hline Expl var (\%) & 0.95 & 0.95 & 0.92 \\
\hline Reliability &
\end{tabular}

Table 2: Factor loadings

Table 3 summarises correlations (in normal fonts) and squared correlations (in italic fonts) between factor z-scores. It is evident that a degree of similarity between Factor 1 and Factor 2 is $23 \%$ and that a degree of similarity between Factor 1 and Factor 3 is 21\%. However, surprisingly, Factors 2 and 3 are not similar (10\%).

\begin{tabular}{|l|l|l|l|}
\hline & Factor 1 z-score & Factor 2 z-score & Factor 3 z-score \\
\hline Factor 1 z-score & 1 & 0.23 & 0.21 \\
\hline Factor 2 z-score & 0.48 & 1 & 0.10 \\
\hline Factor 3 z-score & 0.46 & 0.32 & 1 \\
\hline
\end{tabular}

Table 3: Correlation between factor z-scores

\section{Research Findings-The Three Personas}

\subsection{Persona 1: The outgoing connector}

Persona 1 consists of fiveyoung adults who have been working in different industries, including two marketers, one accountant, one IT intern and one vet intern. Four of them are males, and the average age of this persona is 24.2. This persona has an eigenvalue of 2.5 and explained variable of 19 per cent.

These users in Persona 1 stand out from the rest with their high concerns about establishing relationships with other people, especially those they met offline $(7:+4 ; 11:+2)$. This, of course, includes connecting with family members and close/ old friends $(1:+6 ; 9:+5)$. They appear to like making new friends online $(10:+1)$, but the statement was ranked at +1 which may indicate low interest. Finding dates (2: -2) was also seen not important, yet interestingly they ranked higher on it than the other groups. The statement about "connecting selectively" is ranked significantly lower which further justifies the outgoing personality of these participants. 
Nevertheless, a careful observation could tell that these people feel more concerned about connecting with known acquaintances than strangers.

Given such an outgoing personality, it is consistent to find that they are concerned about SNS having a large number of users $(40:+5)$ and finding more information about people in the real world (46: $+4 ; 48:+3)$. This would support their strong need for establishing relationships. Other than that, they see information about products, companies, celebrities, and events as unimportant (4: $-4 ; 43: 0 ; 47:-4)$. They arealso interested in reading news (49: +3) and sharing resources, especially sharing music and video (5: +4$)$. One participant said that he enjoys sharing clips about outing events with his friends on Facebook. Overall, they do not seem to care very much about Facebook's usability $(45:+1)$ as they agreed that the interface is simple and can be figured out easily.

These users do not seem to be interested in Facebook's conversational functionality, perhaps because they are more attracted to establishing online relationships with people they know in real life. This is reflected by the low concern about the need to communicate with public audiences (29: -2). The only statement being ranked higher than the other groups is "joining SNS pages of common interests" (41: +2$)$, but it was explained by one participant that hejoined groups for gaining knowledge of specialised topics rather than socialising. In this sense, joining pages of interests is more or less similar to reading news. It is also worth noticing that receiving approvals from friends is of high concern to this persona (37: +2$)$. One participant actually explained that the reason and motivation for blogging his opinions about a general event (15: 0 ) are to portrait himself to his friends as being up-to-date. These users are not concerned about trusting others online (16: - $1 ; 22: 0$ ), as one discussed that he would want to be constantly aware of people whether or not they are his friends.

These users' views about privacy threats are unique among the three personas (20: -4$)$. They feel concerned more about the possibility of having their profiles checked by potential job recruiters as well as the risk of losing jobs due to complaints (38: $+1 ; 39$ : 0 ). However, these two statements were ranked at the middle of the sort, and two participants discussed that they would limit their employers' access to their profiles, which together suggest these statements may indicate neutrality. They feel concerned about expanding professional networks on Facebook (8: +3). Furthermore, they do not care about the threats of insult and racism (31: -5; 35: -6) because they can block inappropriate content, whereas insults are perceived as unimportant unless they come from their friends.

Regarding other threats, they generally do not care much about their information being collected by firms (42: -3; 50: -3) or misused (24: -3; 33: -1). This is mostly due to these users believing that they do not post important information in their accounts (13: -1 ; 19: 0 ); therefore, they do not have to be worried about what would happen to their information. Similarly, they are also not bothered by being located offline which links to the threat of cyberstalking (30: -2; 34: -2). One participant reported not usually "checking in" at places while the other said that even if he were tagged by his friends' check-ins, it would not be threatening as long as his home address is not revealed. Again, the emphasis on the nature of the information is displayed here. These users are also not concerned about the threats of scams or trickery (32: -2; 36: 0), which two of them expressed confidence in not getting themselves caught in such schemes. Such beliefs may explain why they do not see much importance in whether Facebook has multiple privacy settings as well as the settings' usability (26: $+1 ; 44: 0)$. In fact, one participant shared that as long as Facebook has underlying privacy protection, he would not care about how easy it is to configure the settings. This user said that he trusts SNS's protection $(23:+1)$, but believed it would be more difficult for local users to seek support from a global company such as Facebook.

\subsection{Persona 2: The reserved trustor}

There are five participants representing Persona 2, among which four are female. All participants were higher education students, and were 21 years old on average. Persona 2 has an eigenvalue of 2.2 and explained variance of 17 per cent. 
The users of Persona 2 appear to contradict much those of Persona 1 as discussed previously. To begin with, they are interested in finding information about people at school or workplace $(46:+5)$ and events (e.g. technology expos and on-sales events), as well as products or companies (e.g. browsing online shop's catalogue) $(4:+4 ; 43:+2)$. Some reported to have followed fan pages of singers but only for getting updates of the latest songs (47: 0), which explains the rank of this statement being at zero point. They also enjoy reading news from multiple sources (49: +3) like the users in Persona 1.

These users are concerned about keeping their networks really personal and intimate. This argument is supported by the high rankings of the statements about keeping in touch with old friends, family members, or close friends $(1:+5 ; 9$ : +6$)$. To let their friends search for them more easily and perhaps to reciprocate trust, these participants feel particularly concerned about using real information to create their Facebook accounts (6: $+3,13:+2)$. More important, their approach to establish relationships is different to those in Persona 1 in that they see connecting selectively as more important $(12:+3)$, and they display a strong disinterest towards people whom they just met physically ( $7:-6 ; 48: 0)$. One participant emphasised that his friends on Facebook are those to whom he had talked in real life and remembers who they are, and having met in-person is not the only criteria to make one become his friend. Of course, finding dates on an SNS (2: -5) is unimportant and untrustworthy to these participants. Extending business networks on Facebook (8: +1$)$ is also not much concerning because they believed that it would be better to do it in-person.

In terms of their interaction preferences, building online trust is ranked highest in this persona among the three (16: $+2 ; 22: 0)$, which indicates the unique concerns regarding this aspect. It is indeed consistent to the overall personality of these users who feel more inclined to keep their networks personal. Joining pages of common interests is also concerning to these participants (41: +2 ), as one of them said that she joined her university's class Facebook page to discuss assignments and share materials. On the other hand, they do not care much about writing blog as those of Persona 1 do (14: -2; 15: -1). In fact, they are not concerned about gaining any approval from friends (37: -2). Furthermore, the need to make their accounts appear good is ranked higher than other personas (3: 0) yet at the neutrality point of the sort. One participant discussed that he is not concerned about that because it is more important for his friends to accept him as who he is regardless of the content that makes up his profile. They view sharing music and video as important $(5:+4)$, and more interestingly, one participant sees this activity as a mean to express subtly her feelings rather than to socialise. However, they are not interested in playing games on Facebook (28: -4) because of several reasons including slow connection, and many games require the users to wait in real time for more ingame "energy" to continue playing. This suggests that they consider gaming as purely for entertainment, and socialising with a larger audience in the game environment may also not be of these users' main interest. In overall, good usability of Facebook $(45:+4)$ is important to them, and one participant admitted that he is quite "low-tech".

Scams and trickery such as fake charity events or hacking accounts and disguising as friends to ask for mobile phone's top-up are relatively concerning to these users (32: +3). Despite being ranked at zero position, these users' concern about the threats of insulting and threatening on Facebook is still higher than the other groups (31; 0). One participant mentioned that after strangers on Facebook gave inappropriate comments on her post about her family issues, her family members complained to her that she should not have brought personal matters online. This left her a negative experience, and she believed such incidents would not happen again in the future. Another participant in this persona admitted to having insulted people on Facebook when he was in high school, and discussed that the impact of such cyber-bullying would not leave much damage. However, he admits not being able to deny that online insulting is happening. In the first case, the female student displayed confidence in not repeating the incident, but she also expressed concern about the threats should they happen again in the future. The male student in the second case stated his concern about the existence of cyberbullying, yet he rejected the severity of his own bullying in the past. Both cases suggested that concerns about these threats are conditional (e.g. feel confident because having learnt the 
lesson but concerned if the insulting actually happens again; feel concerned about cyberbullyingin general but unconcerned about own action). This could explain why the participants ranked the statement in the middle of the $\mathrm{Q}$ sort, which gives a tentative and unsure answer about their concerns.

The threat of cyber-stalking is ranked as not concerning (34: -1), despite three participants were fully aware of such problem. In particular, two of them reported to have friends who were cyber-stalked and had their pictures modified and publicly teased on Facebook. Similarly, the other user said that she felt terrified when read news about a high school student who also had pictures ridiculed online and committed suicide because of that. Nevertheless, perhaps because these participants did not expect such situation would happen to them so they ranked this threat as unconcerned. In fact, one of them discussed that she accepts her pictures being publicly visible to the public, which could explain why the need for sharing personal photos to public audience was viewed as less concerning (25: -2). In contrast with participants with Persona 1 that worry about having their contents seen by employers (38: -4; 39: - 3), these users do not display concerns about those threats. One participant believed recruiters would not do that while the other discussed empathetic employers would not misunderstand the staff's online actions. Likewise, the threats of having personal information misused or shared by organisations (24: -3 ; 42: -2 ) are also not an issue as one user argued that her information (only contains name and school) that if leaked would not bring her any harm in any way.

\subsection{Persona 3: The threats perceiver}

Persona 3 consists of three participants having different genders and occupations. More specifically, there are two females and one male. They are higher education student, HR executive and IT auditor respectively. The average age of Persona 3's participants is 24 years old. The factor has an eigenvalue of 1.7 and explained variance of 13 per cent.

The users of Persona 3 differentiate themselves from the rest with their unique concerns about establishing relationships, SNS functionalities, and privacy threats. In particular, they saw expanding professional networks $(8:+4)$ as most important. They also share high concerns about connecting selectively with friends and family members $(1:+3 ; 9$ : $+6 ; 12$ : +3$)$ as compared to the other personas. They also value connections with people whom they met inperson (7: +3) rather than online (10: -3; 29: -5). Their need to connect selectively (perhaps with business contacts) is supported by the low concern about being able to connect with everyone met in real life (11).

Finding information (4: -3 ; 43: -2 ; 46: $+1 ; 47:-3$, 48: 0 ; 49: -2 ) is not these participants' main purpose. One participant complained that her Facebook's feeds contains a mixture of some actual news and many stories and pictures that are not credible and inadequate (49: -2), while another said that they would rather go to official websites to search for information about events or products (4: $-3 ; 47$ : -3$)$. Similarly, these users care the least about making their accounts appear good (3: -5), yet feel quite concerned about receiving approval from friends $(37:+2)$. All of them agreed that they do not want to tailor too much or fake their profiles to make themselves more attractive, and one participant discussed that approvals are only valuable when coming from their realistic features on their profiles. They also display disinterest towards sharing sensitive information such as gender preferences or drinking habits (18: -4), which one participant explained that such information hold no value for building their images. Likewise, they are also not interested in sharing or joining discussions about religions or politics (27: -2), as they explained that people are entitled to their own opinions and debating these topics may result in legal issues. If there is a need to share anything, one participant explained that he would write blogs and set it available only to him $(14:+1 ; 15: 0)$. In this case, a personal blog is seen as a tool to release stress.

Not exposing much information on Facebook is slightly concerning (19: +1). One participant especially feels concerned about relationship status because they would cause gossip. This may relate to their uniquely high concerns about the privacy threats. Among these participants' top concerns is the belief that their privacy being threatened $(20:+5)$, followed by scams and trickery $(32:+5)$. They also told the fear of having their children's photos misused on Facebook, 
as well as scams that attempted to collect profile's data for marketing purposes, trick them into a pyramid scheme or request top-up for mobile credit by using their friend's hijacked account. Two participants advised their friends to contact them if received strange requests from their account. These viewpoints are related to the high concern about identity theft and cyberstalking $(24: 0 ; 33:+2,34:+2)$, which they believe the main purpose is for gathering information about their behaviours and friends circle so that the stalker may craft realistic trickery (e.g. cloning their accounts). On the other hand, they are not concerned about having locations displayed on Facebook as they explained one could stay at home and check in at somewhere else, and this viewpoint is similar to those in Persona 2.

These participants also feel concerned about having their information collected and shared by organisations, including Facebook (42: $+2 ; 50: 0)$. However, as much as they value business networks on SNS, they are not bothered by the risk of having profiles checked by recruiters and especially losing jobs because of inappropriate contents (38: -2; 39: -4). A participant who was an HR executive shared that she does not usually screen candidates using social media profiles but rather calls and checks their references. The other participants reported they had never complained on Facebook, and if they did such a thing, their friends would come to their rescues. Another also argued that the employers would care more about the staff's abilities rather than what they post on Facebook. Again, this explanation is similar to one of Persona 2's users.

In response to the high concerns about the privacy threats, these participants see Facebook having multiple privacy settings with good usability as important $(26:+4,44:+3)$, yet they do not feel concerned about trusting its protection (23: 0). One of them expressed that he would generally trust Facebook's protection, but feels unsure what Facebook would do to help him if his account got stolen. On the other hand, building online trust is unreliable thus unimportant to them (16: -1; 22: -1). They justified that it is important to maintain being careful with the others, especially when their friends' accounts could be faked any time.

\subsection{Summary of the three personas}

We summed theranks of the items grouped by their respectivedomains, then normalised those summations by the number of items so to create a radar chart (Figure 3) that compares the three personas against each other. At first glance, Persona 1 has the most concerns about Relationships and SNS functionalities domains, and cares the least about privacy threats. On the other hand, Persona 2 contradicts the concerns of Persona 1 and focuses on finding information. Persona 3 appears to be a combination of personas 1 and 2 , especially in terms of their concerns about Relationships and SNS functionalities. However, they care the most about Privacy threats and the least about Interactions and Finding information. These brief visual analyses are consistent with the previous within-persona discussions. 


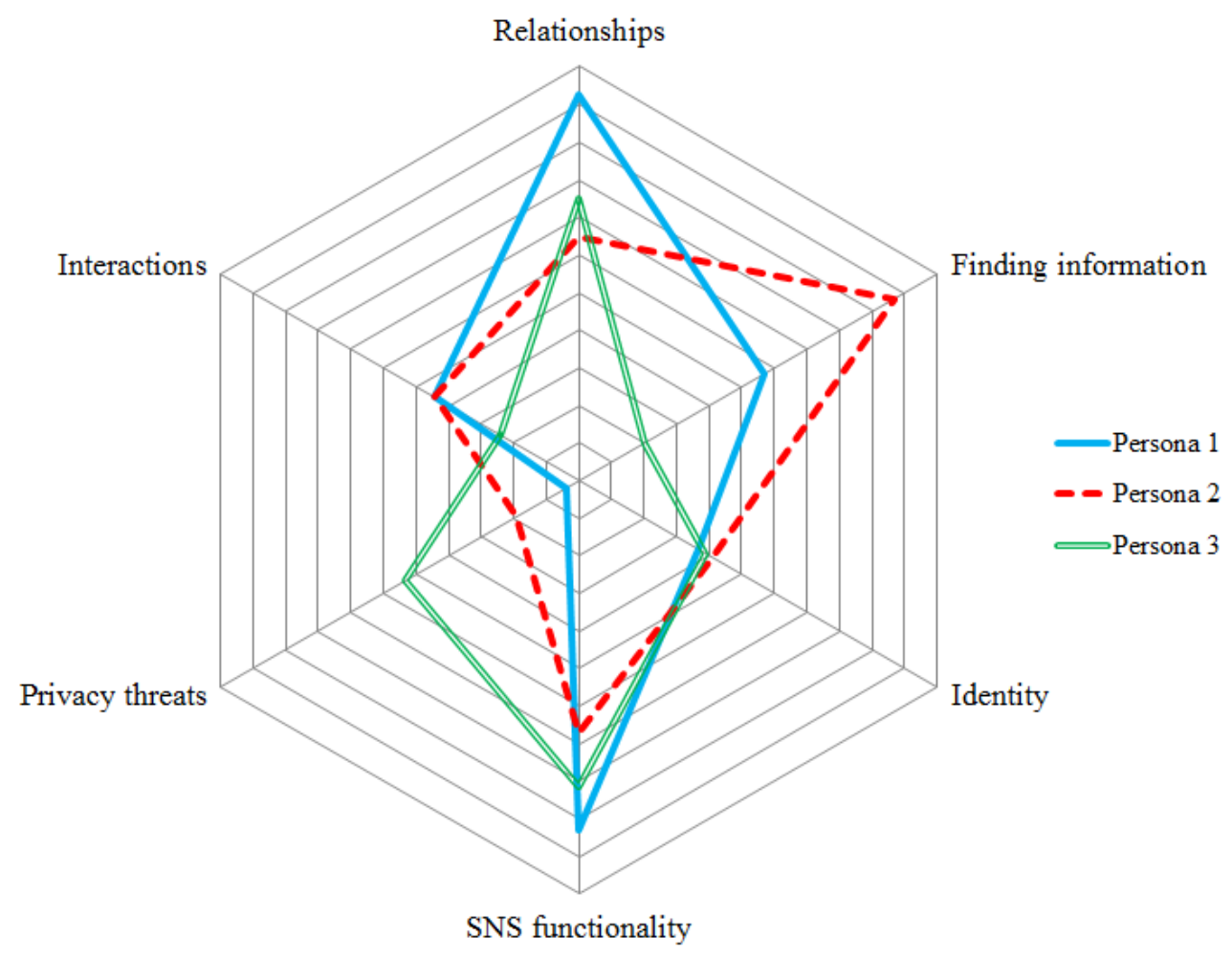

Figure 3: Summary of three personas

First, Persona 1-- the Outgoing Connector-- is interested in establishing relationships with a stronger alignment towards known acquaintances and business contacts in offline world rather than online. To achieve this goal, their connection strategy is quite unselective and reflects their highest concerns about Relationships domain. They are interested much in finding information about people rather than events or products. Furthermore, they also care the most among the three personas about Facebook having a large number of users, which resulted in their highest concerns about SNS functionalities. One explanation provided by a Persona 1's participant was that the amount of users is associated with the number of online threats. However, another reason behind this concern could be to ensure a greater chance for connecting with the people of their interest, especially in the offline world. The users of Persona 1 appear to be most confident in handling the privacy threats on Facebook, which resulted in their lowest concerns regarding those threats as compared to the other personas. In fact, these participants reported to know well how to limit others' access to their profiles as well as block the undesired contents.

Second, Persona 2-- the Reserved Trustor--pays attention to information about not only people at school but also products, organisations, and events. Consequently, this broad and intensive attention to information made them having the highest concerns regarding this domain. Unlike Persona 1, these participants' connection approach is selective, and meeting physically alone does not make that person become their online friend. This subsequently explains their slight interest in establishing business network on Facebook, which they argued that it should be better done in-person. They also joined Facebook pages to exchange information as Persona 1 does, except that those pages are university or class's online communities that consist of known acquaintances only. They also do not care about gaining social approval as much as personas 1 and $3 \mathrm{do}$. One participant actually expected his trusted circle to accept him as who he is, and sharing music and video was discussed as the mean to express feelings by another participant. After all, building trust on Facebook is highly concerning to Persona 2. These female students have a higher concern about the threats of scams and trickery on Facebook than the other two personas, which may reveal them as the specific threats that these students encounter. Furthermore, they have a more optimistic 
expectation than Persona 1 that their employers would not judge the profile's contents. However, it is worth mentioning that these users do not have work experience like those of personas 1 and 3, which may make their expectations unrealistic.

Third, Persona 3- - the Threats Perceiver- - appears to be a mixture of both personas. To start with, they resemble thosein Persona 1 that they are interested in connecting with offline people and especially for expanding professional network. However, they prefer to connect selectively like Persona 2. This could explain why they do not feel concerned about making their accounts appear good, and friends' approvals only matter if they are about the true and real information on these users' profiles. Unlike the other personas, finding information is not Persona 3's purpose in using Facebook. They especially displayed concerns about the credibility and appropriateness of the information on Facebook. More interestingly, Persona 3 has the most concerns about privacy threats. Perhaps these concerns explain their selective and reserved connection approach, unlike Persona 2 which would be based on trust and intimacy. Persona 3 feels bothered by the belief that their online privacy is threatened, which includes threats such as identity theft, cyber stalking, scams and trickery. In contrast to Persona 1, Persona 3 does not feel concerned about the threats of losing job and having recruiters check for inappropriate contents. Finally, they also have higher interests in Facebook having multiple privacy settings and their usability.

\section{Discussion}

\subsection{Concerns about finding information}

Finding and using information have been supported to be one of the core activities on SNS (Lampe et al. 2006), especially for establishing relationships (Boyd and Ellison 2007; Ellison et al. 2011). Our results are consistent with prior studies as the concerns about finding information on Facebook (i.e. "social searching") were ranked relatively high. More importantly, we detected dissimilarities in these concerns and anticipate that they may be associated with the participants' different demographics as well as other concerns. While the working, male-dominant Persona 1 was interested in finding information about anyone they met offline, especially for making new business connections, the female students in Persona 2 only felt concerned about those at school and prioritised information about products, events and parties instead. In fact, this reflected the study of Cha (2009) which found a positive relationship between female gender and attitude towards shopping on SNS, based on their survey of 167 college students in the US whose average age was 20.71 years old. On the other hand, a possible explanation for the prior result is that the participants in Persona 1 who have been working realised a greater need to expand their network, while Persona 2's students do not have much capability to reach professional contacts. All users of Persona 3 questioned the credibility and appropriateness of information sources on Facebook, as much as being critical about privacy threats. While they did not explain their disinterests in finding information in general, the attributes of information on Facebook could determine the users' motivation to find and use them. Additionally, the lack of interest in finding information about other people could also be due to the fact that their connections had been carefully selected and established as a result of their offline investigations.

\subsection{Concerns about identity}

Establishing and maintaining online identities is another major topic in SNS research (Boyd and Ellison 2007). Our findings support that the three personas expected their profiles' information (e.g. real names and pictures) to be searched by friends (Lampe et al. 2006), and some intentionally included identifiable information for that purpose while accepting the privacy risks. Unlike the recent research by Karl et al. (2010) which surveyed US and German students about sensitive and private contents on Facebook such as gender preferences and drinking habit, theyoung adults in Vietnamese context consistently demonstrated disinterests in posting or joining discussions about those topics on Facebook. Interestingly, the recent research by Nadkarni and Hofmann (2012) hypothesised that individualistic cultures would disclose more private information and more likely to promote controversial topics than 
collectivistic ones. As the sample of our study consists of Vietnamese whose culture is collectivistic (Hofstede 2001), this finding may be an indicator that supports that hypothesis.

Self-presentation and self-promotion (Boyd and Ellison 2007; Kietzmann et al. 2011; Krasnova et al. 2010; Mehdizadeh 2010) were also examined in our study. The three personas did not feel concerned about making their accounts appear good, whereas receiving approvals from the others was more concerning. Most participants understood the prior statement as intentionally using enhanced photos and desirable information to the extent of presenting fake information, and they opposed against such activities. On the other hand, participants of personas 1 and 3 admitted to feel concerned about receiving friends' approvals, whereas Persona 2 saw it as not concerning. This suggests self-presentation may not only be about the different needs to display desirable traits, but also depend on the existing relationships which subsequently characterise the target audiences. Furthermore, discussed findings about a Persona 1's participant who sought knowledge and portrayed himself as being up-to-date by blogging his opinions imply that certain types of self-presentation are more accepted than the others.

\subsection{Concerns about interactions and Facebook's functionalities}

Interactions on Facebook are reflected in activities such as broadcasting messages via writing blogs or participating in common environments such as online games or social pages (Ellison et al. 2011; J oinson, 2008; Kietzmann et al. 2011). On the other hand, Facebook facilitated these activities with its functionalities, thus we discuss the concerns of these two domains together in this section. J oining groups on Facebook was categorised in the theme of "making new contacts" by J oinson (2008), and it was discussed to find people with common interests by Ellison et al. (2011). However, participants in both personas 1 and 2 did not mention about socialising on those pages but rather joined for exchanging information and acquiring knowledge. This is supported by the low concern about wanting to communicate with the public audience displayed among the three personas. Furthermore, combined with the low concerns about finding dates, these findings suggest that Facebook may not be suitable for making new contacts.

As discussed previously, online trust was not highly concerned by most participants, except Persona 2. Even so, all three personas held a common belief that they could not simply trust online profiles easily, which also includes those that belong to their friends. A possible explanation is that for Persona 1, their trusting stance is high in nature since they are outgoing and like to make new connections online, thereby not being concerned over a lack of trust; while for Persona 2, they aim to make connections with either professionals or those met in real life, which also makes them feel less worried about untrustworthy people. As a consequence, this questions the socialising purpose of gaming and joining pages of common interests on Facebook. Likewise, a participant of Persona 3 explained writing blogs as a tool to relieve stress, and the blog's availability was set to private and available only to the author. On the other hand, Persona 2 provided an example where a participant subtly expressed her moods and feelings by sharing songs and video. In these cases, writing blogs and sharing music and video appear to have more purposes than simply broadcasting the messages.

\subsection{Concerns about privacy threats}

The different concerns about privacy threats distinguished the three personas. Overall, personas 1 and 2 hold much fewer concerns about privacy threats as compared with Persona 3. Between Persona 1 and the others, the prior is bothered more by the threat related to occupations while the other two personas have higher concern about scams and trickery. By considering the demographics of Personas 1 and 2, it could be hypothesised that the types of concerned privacy threats are associated with the differences in occupations if not even genders. Nevertheless, occupational threats were also not consistently concerning among the working groups as it was shown in the comparison between Personas 1 and 3 . We believe such dissimilarities could be explained by the different approaches in establishing relationships, which Persona 1 appears to connect with wider networks than Persona 3. As a consequence, 
Persona 1 may be aware of their larger exposure to the threats of having careers affected by inappropriate contents or having their profile checked by recruiters.

Among the three personas, Persona 3 holds the highest concerns about their online privacy being threatened by a wide range of threats mentioned in previous studies (Krasnova et al. 2010, 2009; Stutzman et al. 2011). Moreover, these participants may have the attributes of the users who are aware of third parties' violation of privacy, which would normally be invisible to other "average users" (Debatin et al. 2009). The varied concerns about one's online privacy being threatened may also be accounted by the different perceived values of information. In fact, most participants of Personas 1 and 2 who had little concern about this aspect agreed so, and one even explained that her information being leaked would not bring severe consequences. Interestingly, this conjecture was supported by the three personas' consistently low concern about being located physically by check-ins. A majority of participants discussed that virtual check-ins could be easily faked (i.e. not checking in at actual locations). Consequently, they argued that such information does not hold much meaning and would result in little harm. On the other hand, the higher concern about cyber-stalking was tied to the information in their profiles such as school or home addresses, rather than checked-in places.

While the threat of spam and spim is evolving and migrating from the traditional email to social media platforms (Symantec 2014), there was not much concern about this threat. Some participants noticed the increased amount of spam received since they joined Facebook, but were also unsure whether SNS was the cause. Nonetheless, they reported to either set up spam filter or register Facebook's account with unimportant mailboxes to mitigate the problem. Additionally, we considered racism as a possible social threat on SNS, but all personas displayed no concern about this threat and denied its existence. On the other hand, the threat of insult and threatening was ranked consistently at a higher position, and the concern about it was discussed as a yes-and-no answer. As presented in the findings' sections, some participants revealed that they had experiences with this threat before and now believed it would not happen again, whereas others shared that it is not concerning as long as the insults do not come from their friends. This finding reflects the medium concerns regarding social threats as surveyed in the study by Krasnova et al. (2009).

\subsection{Concerns about relationships}

Keeping in touch with friends and family members have been commonly discussed by prior research (Ellison et al. 2011; Park et al. 2009; Subrahmanyam et al. 2008). Our study also found maintaining these relationships is of consistently high concern to the three personas' participants. In addition, the users of Personas 1 and 3 who had work experiences were concerned about expanding their professional networks. This finding reflects the discussions in relevant studies (A. Carrillat et al. 2014; Dimicco and Millen 2007; Skeels and Grudin 2009) that argued Facebook is becoming more related to organisational aspects such as business networking and recruitment. Furthermore, two distinct networking approaches were detected from these personas even though they share similar demographics. Specifically, Persona 1 is more interested in connecting with everyone met offline and online, whereas Persona 3 only feels concerned about connecting selectively with people met in person.

\section{Implications and Limitations}

We believe our typology of Facebook personas and their specific concerns hold practical and theoretical implications. The findings could be helpful for designing marketing tactics and for social media developers, especially Facebook, to enhance user's experiences. While our finding about the relationships between demographics (i.e. genders and occupations) and their information of interest (i.e. about people or products/ events) cannot be generalised to a larger population, it was consistent with the result of a prior research in the US context about young female students having a positive attitude towards online shopping on SNS. The female students of Persona 2 were highly concerned about building online trust and connecting with known acquaintances. It may be worthwhile to explore whether referrals and testimonials 
based on marketing would be effective for the demographics of this persona. On the other hand, finding information about celebrities, as well as events (e.g. expos and sales events) and parties, was ranked uniformly low by personas 1 and 3 . While this could be due to these personas' preferences, it could also hint that Facebook pages may not be the best platform to disseminate these types of information to the Vietnamese young adult audiences.

The high level of concern about "connecting with acquaintances" supports this item to be the main purpose of using Facebook. Furthermore, previous analyses on the low ranking items such as "finding dates" and "connecting with everyone online" suggest that Facebook may not be a suitable platform for connecting with strangers. On the other hand, expanding business network on Facebook was highly ranked by participants with work experiences. While there are other SNS that are exclusively designed for professional networks such as LinkedIn, perhaps Facebook with its unique socialising functionalities would enable different types of interactions that may be interesting for future research to explore. For example, LinkedIn focuses on effectively encapsulating and showcasing its users' work experiences, which are most suitable for disseminating profiles and reaching new people. On the other hand, users on Facebook would exploit the interactional functions to expand their business networks via richer communication, or perhaps by showcasing their desirable traits to potential contacts in a different way.

In addition, social pages and blogs are the interactional tools that are available on Facebook for its users to socialise with people of common interests and exchange information. However, some users shared that they joined social pages on Facebook mainly for obtaining information rather than socialising. Perhaps organisations that run Facebook pages may want to focus more on delivering the desired content to the audience, as well as control the types of information and sources on their page that would attract the users' attention (e.g. other users' comments). Moreover, we also found multiple ways to express feelings from the users. For instance, a female student from Persona 2 reported to communicate her feelings by sharing music and video, whereas a more matured user from Persona 3 chose to write down thoughts in his blog and set it available only to him as a way to relieve stress. Consequently, we learned that Facebook's functions may not always be used for their original purposes and could contain different meanings.

We also found the participants to hold different views about their online privacy. The three personas seemed to understand well which information would be appropriate to be disclosed, and some intentionally accepted the risks to achieve their purposes (e.g. included identifiable information to make accounts searchable by friends). Our sample's participants also considered the threat of racism as not existing, which may be due to the fact that Vietnam's population is generally homogeneous or that they have a high level of racism tolerance. As a result, it would be interesting to replicate this research in heterogeneous and multicultural countries (e.g. Australia) and compare the findings. On the other hand, the concerns about usability of Facebook's multiple privacy settings were relatively high in general. Moreover, participants of Persona 3 especially feel concerned about the privacy threats on Facebook. While these participants could have many reasons and past experiences that led to such high concerns, it may also be due to them not knowing much about the available privacy settings. SNS should continue to educate their users as well as make it easy for them to effectively use the provided protection. Furthermore, a majority of our participants felt unsure about whether Facebook as a global organisation would provide them assistance if their online privacy was harmed, provided that they are local users. In this case, the perceived presence of SNS organisations would play a crucial role in providing the global users a state of security and may subsequently affect their behaviours, thus its visibility should be enhanced.

Even though we have attempted to ensure rigour and richness in analysis and discussion, our research inevitably contains limitations. First, the proposed typology of the three personas is exploratory and we cannot confirm that their unique concerns had not occurred by sampling's chance. Secondly, 49\% of explained variance stems from the small sample size of 14 participants, and there may be more profiles that are not discovered in this study. 
Our analysis suggested three as the optimum number of unique personas that could be extracted. With a larger number of participants and items, there could be more different personas to be found. These limitations, combined with our focus on Vietnameseyoung adults, clearly need follow-up studies in the future. In that case, this typology may serve as the first step to classify Facebook's young adult users, and it may be changed as being tested by future studies in larger samples or different contexts. More importantly, the items used to construct this typology would be especially useful for developing future research instruments and bring forward an important theoretical implication.

One of the theoretical implications includes the consensus and distinct items as shown in the Appendix. The consensus items can be used to develop an invariant measurement which is stable across all personas and can be subsequently tested with factor analysis. Researchers can use the distinct items to develop a measurement to classify users into different personas that can be tested with latent class analysis, since each item can be used to capture specific traits of each persona. In addition, our study also demonstrated a new way to classify social media users' personalities by using Q-methodology. The virtual world on SNS such as Facebook is indeed multifaceted and consists of diverse end users' concerns and behaviours. We look forward to seeing our practical and theoretical implications contribute to future studies that further expand our knowledge of this vast research domain.

\section{References}

A. Carrillat, François, Alain d'Astous, and Emilie Morissette Grégoire. 2014. "Leveraging Social Media to Enhance Recruitment Effectiveness." Internet Research, 24 (4): 474- 495.

Boyd, Danah M., and Nicole B. Ellison. 2007. “Social Network Sites: Definition, History, and Scholarship." J ournal of Computer-Mediated Communication, 13 (1): 210- 230.

Brandtzaeg, Petter Bae, and J an Heim. 2011. "A Typology of Social Networking Sites Users." International J ournal of Web Based Communities, 7 (1): 28.

Cha, Jiyoung. 2009. "Shopping on Social Networking Web Sites.” Journal of Interactive Advertising, 10: 77-93.

Debatin, Bernhard, J ennette P. Lovejoy, Ann-Kathrin Horn, and Brittany N. Hughes. 2009. "Facebook and Online Privacy: Attitudes, Behaviors, and Unintended Consequences." J ournal of Computer-Mediated Communication, 15 (1): 83- 108.

Dimicco, Joan Morris, and David R Millen. 2007. “Identity Management: Multiple Presentations of Self in Facebook." In GROUP'07, 0- 3.

Ellison, N. B., C. Steinfield, and C. Lampe. 2011. “Connection Strategies: Social Capital Implications of Facebook-Enabled Communication Practices." New Media \&Society, 13: 873- 892.

Facebook. 2015. “Company Info.” Newsroom. http:// newsroom.fb.com/ company-info/ .

Gross, Ralph, and Alessandro Acquisti. 2005. "Information Revelation and Privacy in Online Social Networks." In ACM Workshop on Privacy in the Electronic, 71- 80.

Hassouneh, Diana, and Malaika Brengman. 2014. "A Motivation-Based Typology of Social Virtual World Users." Computers in Human Behavior, 33: 330- 338.

Hofstede, Geert. 2001. Culture's Consequences: Comparing Values, Behaviors, Institutions, and Organizations Across Nations. Second Edi. Thousand Oaks CA: Sage Publications.

J oinson, Adam N. 2008. “'Looking At', 'Looking Up' or 'Keeping up With' People? Motives and Uses of Facebook.” In CHI 2008, 1027- 1036.

Karl, Katherine, J oy Peluchette, and Christopher Schlaegel. 2010. “Who's Posting Facebook Faux Pas? A Cross-Cultural Examination of Personality Differences." International J ournal of Selection and Assessment, 18 (2): 174- 186. 
Kietzmann, Jan H., Kristopher Hermkens, Ian P. McCarthy, and Bruno S. Silvestre. 2011. "Social Media? Get Serious! Understanding the Functional Building Blocks of Social Media." Business Horizons, 54 (3): 241- 251.

Krasnova, Hanna, Oliver Günther, Sarah Spiekermann, and Ksenia Koroleva. 2009. "Privacy Concerns and Identity in Online Social Networks." Identity in the Information Society, 2 (1): 39- 63.

Krasnova, Hanna, Sarah Spiekermann, Ksenia Koroleva, and Thomas Hildebrand. 2010. “Online Social Networks: Why We Disclose." J ournal of Information Technology, 25(2): 109- 125.

Lampe, Cliff, Nicole Ellison, and Charles Steinfield. 2006. "A Face (book) in the Crowd: Social Searching vs. Social Browsing." In Proceedings of the 2006 20th Anniversary Conference on Computer Supported Cooperative Work. ACM, 167- 170.

Mehdizadeh, Soraya. 2010. "Self-Presentation 2.0: Narcissism and Self-Esteem on Facebook." Cyberpsychology, Behavior and Social Networking, 13 (4): 357- 364.

Meyen, M., S. Pfaff-Rudiger, K. Dudenhoffer, and J. Huss. 2010. "The Internet in Everyday Life: A Typology of Internet Users.” Media, Culture \& Society, 32 (5): 873- 882.

Nadkarni, Ashwini, and Stefan G Hofmann. 2012. “Why Do People Use Facebook?” Personality and Individual Differences, 52 (3): 243- 249.

Park, Namsu, Kerk F Kee, and Sebastián Valenzuela. 2009. "Being Immersed in Social Networking Environment: Facebook Groups, Uses and Gratifications, and Social Outcomes." Cyberpsychology \& Behavior : The Impact of the Internet, Multimedia and Virtual Reality on Behavior and Society, 12 (6): 729- 733.

Pew Research Center. 2014. "Demographics of Key Social Networking Platforms.” Social Media Update 2014. http:// www.pewinternet.org/2015/01/09/demographics-of-keysocial-networking-platforms-2/ .

Pittayachawan, S, M Singh, and Brian Corbitt. 2008. "A Multitheoretical Approach for Solving Trust Problems in B2C E-Commerce." International J ournal of Networking and Virtual Organisations, 5: 369-395.

Skeels, Meredith M, and J onathan Grudin. 2009. "When Social Networks Cross Boundaries: A Case Study of Workplace Use of Facebook and LinkedIn.” In GROUP'09, 10:95- 103.

Stephenson, William. 1953. "The Study of Behavior: Q-Technique and Its Methodology."

Stoughton, J William, Lori Foster Thompson, and Adam W. Meade. 2013. "Big Five Personality Traits Reflected in J ob Applicants' Social Media Postings.” Cyberpsychology, Behavior and Social Networking, 16 (11): 800-806.

Stutzman, F. 2006. "An Evaluation of Identity-Sharing Behavior in Social Network Communities." International Digital and Media Arts J ournal, 3: 10- 18.

Stutzman, Fred, Robert Capra, and J amila Thompson. 2011. "Factors Mediating Disclosure in Social Network Sites." Computers in Human Behavior, 27 (1): 590- 598.

Subrahmanyam, Kaveri, Stephanie M Reich, Natalia Waechter, and Guadalupe Espinoza. 2008. "Online and Offline Social Networks: Use of Social Networking Sites by Emerging Adults." J ournal of Applied Developmental Psychology, 29 (6): 420- 433.

Symantec. 2014. “Internet Security Threat Report 2014.” Vol. 19. http:// www.symantec.com/ content/ en/ us/ enterprise/ other_resources/bistr_main_report_v19_21291018.en-us.pdf.

Tan, Xin, Li Qin, Yongbeom Kim, and J effrey Hsu. 2012. “Impact of Privacy Concern in Social Networking Web Sites." Internet Research 22, (2): 211- 233. 
Watts, S., and Stenner, P. 2012. “Doing Q Methodological Research: Theory, Method and Interpretation." Qualitative Research in Psychology, 2 (1): 67- 91.

WeAreSocial. 2012. "Social, Digital and Mobile in Vietnam." http:/ / wearesocial.sg/blog/ 2012/ 10/ social-digital-mobile-vietnam-oct-2012/ .

WeAreSocial. 2014. "Social, Digital \& Mobile in APAC in 2014." http:// wearesocial.net/ blog/2014/01/ social-digital-mobile-apac-2014/ .

WeAreSocial. 2015. "Digital, Social \& Mobile Worldwide in 2015." http:/ / wearesocial.net/ blog/2015/ 01/ digital-social-mobile-worldwide-2015/ .

Zabala, Aiora. 2014. “Qmethod: A Package to Explore Human Perspectives Using Q Methodology." 


\section{Appendix}

\begin{tabular}{|c|c|c|}
\hline Domains & \# & Items \\
\hline \multirow[t]{8}{*}{ Relationships } & 1 & Connect with family or close friends \\
\hline & 7 & Connect with people just met outside \\
\hline & 9 & Keep in touch with old friends \\
\hline & 10 & Can connect with everyone online \\
\hline & 11 & Can connect with everyone met in real life \\
\hline & 12 & Connect selectively \\
\hline & 2 & Find dates \\
\hline & 8 & Expand business and professional network \\
\hline \multirow[t]{7}{*}{ SNS functionalities } & 28 & Want to play games on this SNS \\
\hline & 5 & Share music and video \\
\hline & 26 & SNS has multiple privacy settings \\
\hline & 40 & SNS has large number of users \\
\hline & 44 & Good usability of privacy settings \\
\hline & 45 & Good usability of SNS \\
\hline & 23 & Trust protective service and function of SNS \\
\hline \multirow[t]{7}{*}{ Interactions } & 25 & Want to share personal pictures to public audience \\
\hline & 29 & Want to communicate with public audience \\
\hline & 41 & J oin SNS pages of common interests \\
\hline & 15 & Write blog about general experiences or events \\
\hline & 14 & Write blog about private and personal experiences \\
\hline & 16 & Building trust on this SNS is important \\
\hline & 22 & Trust my friends on SNS will not harm me \\
\hline \multirow[t]{6}{*}{ Finding information } & 46 & Find more info about people at school or workplace \\
\hline & 48 & Find more info about people just met outside \\
\hline & 47 & Find info about celebrities \\
\hline & 43 & Find info about products or companies \\
\hline & 49 & Read news from multiple sources \\
\hline & 4 & Find info about events and parties \\
\hline \multirow[t]{13}{*}{ Privacy threats } & 31 & There can be threats of insult, threatening \\
\hline & 32 & There can be threats of scams, trickery \\
\hline & 33 & There can be threats of identity theft \\
\hline & 34 & There can be threats of cyber-stalking \\
\hline & 30 & Cannot locate me in real life \\
\hline & 35 & There can be threats of racism \\
\hline & 36 & There can be threats of spam or spim \\
\hline & 38 & Threats of recruiters check info on SNS \\
\hline & 39 & Threats of losing job due to complaint or talk bad about job \\
\hline & 42 & Threats of having personal info shared by organisations \\
\hline & 50 & SNS company could use my personal info \\
\hline & 20 & My privacy is threatened on this SNS \\
\hline & 24 & My identifiable info can be used for bad purposes \\
\hline \multirow[t]{9}{*}{ Identity } & 6 & Use real info to create account \\
\hline & 13 & Use real name on this SNS \\
\hline & 17 & Publish my real pictures \\
\hline & 3 & My account needs to appear good \\
\hline & 21 & Create SNSs accounts with similar info \\
\hline & 19 & Not expose much info on this SNS \\
\hline & 18 & Share sensitive info e.g. gender preferences or drinking habit \\
\hline & 27 & Share info about religion or political points of view \\
\hline & 37 & Receive approval from SNS friends e.g. Facebook likes \\
\hline
\end{tabular}

Table A1: List of items (statements) 


\begin{tabular}{|c|c|c|c|c|c|c|}
\hline \multirow[b]{2}{*}{ \# } & \multirow[b]{2}{*}{ Items } & \multicolumn{4}{|c|}{ Distinguishes } & \multirow[b]{2}{*}{ Consensus } \\
\hline & & All & $\begin{array}{l}\text { F1 } \\
\text { only }\end{array}$ & $\begin{array}{l}\text { F2 } \\
\text { only }\end{array}$ & $\begin{array}{l}\text { F3 } \\
\text { only }\end{array}$ & \\
\hline 1 & Connect with family or close friends & $\mathbf{0}$ & - & - & - & - \\
\hline 2 & Find dates & $\mathbf{0}$ & - & - & - & - \\
\hline 3 & My account needs to appear good & $\mathbf{0}$ & - & - & - & - \\
\hline 39 & $\begin{array}{l}\text { Threats of losing job due to complaint or talk bad } \\
\text { about job }\end{array}$ & $\mathbf{0}$ & - & - & - & - \\
\hline 13 & Use real name on this SNS & - & $\mathbf{0}$ & - & - & - \\
\hline 25 & Want to share personal pictures to public audience & - & $\mathbf{0}$ & - & - & - \\
\hline 31 & There can be threats of insult, threatening & - & $\mathbf{0}$ & - & - & - \\
\hline 32 & There can be threats of scams, trickery & - & $\mathbf{0}$ & - & - & - \\
\hline 38 & Threats of recruiters check info on SNS & - & $\mathbf{0}$ & - & - & - \\
\hline 40 & SNS has large number of users & - & $\mathbf{0}$ & - & - & - \\
\hline 48 & Find more info about people just met outside & - & $\mathbf{0}$ & - & - & - \\
\hline 50 & SNS company could use my personal info & - & $\mathbf{0}$ & - & - & - \\
\hline 4 & Find info about events and parties & - & - & $\mathbf{0}$ & - & - \\
\hline 7 & Connect with people just met outside & - & - & $\mathbf{0}$ & - & - \\
\hline 16 & Building trust on this SNS is important & - & - & $\mathbf{0}$ & - & - \\
\hline 18 & $\begin{array}{l}\text { Share sensitive info e.g. gender preferences or } \\
\text { drinking habit }\end{array}$ & - & - & $\mathbf{0}$ & - & - \\
\hline 28 & Want to play games on this SNS & - & - & $\mathbf{0}$ & - & - \\
\hline 37 & $\begin{array}{l}\text { Receive approval from SNS friends e.g. Facebook } \\
\text { likes }\end{array}$ & - & - & $\mathbf{0}$ & - & - \\
\hline 47 & Find info about celebrities & - & - & $\mathbf{0}$ & - & - \\
\hline 5 & Share music and video & - & - & - & $\mathbf{0}$ & - \\
\hline 10 & Can connect with everyone online & - & - & - & $\mathbf{0}$ & - \\
\hline 11 & Can connect with everyone met in real life & - & - & - & $\mathbf{0}$ & - \\
\hline 14 & Write blog about private and personal experiences & - & - & - & $\mathbf{0}$ & - \\
\hline 20 & My privacy is threatened on this SNS & - & - & - & $\mathbf{0}$ & - \\
\hline 24 & My identifiable info can be used for bad purposes & - & - & - & $\mathbf{0}$ & - \\
\hline 29 & Want to communicate with public audience & - & - & - & $\mathbf{0}$ & - \\
\hline 33 & There can be threats of identity theft & - & - & - & $\mathbf{0}$ & - \\
\hline 34 & There can be threats of cyber-stalking & - & - & - & $\mathbf{0}$ & - \\
\hline 41 & J oin SNS pages of common interests & - & - & - & $\mathbf{0}$ & - \\
\hline 42 & $\begin{array}{l}\text { Threats of having personal info shared by } \\
\text { organisations }\end{array}$ & - & - & - & $\mathbf{0}$ & - \\
\hline 43 & Find info about products or companies & - & - & - & $\mathbf{0}$ & - \\
\hline 49 & Read news from multiple sources & - & - & - & $\mathbf{0}$ & - \\
\hline 6 & Use real info to create account & - & - & - & - & $\mathbf{0}$ \\
\hline 9 & Keep in touch with old friends & - & - & - & - & $\mathbf{o}$ \\
\hline 12 & Connect selectively & - & - & - & - & $\mathbf{0}$ \\
\hline 15 & Write blog about general experiences or events & - & - & - & - & $\mathbf{0}$ \\
\hline 17 & Publish my real pictures & - & - & - & - & $\mathbf{0}$ \\
\hline 21 & Create SNSs accounts with similar info & - & - & - & - & $\mathbf{o}$ \\
\hline 22 & Trust my friends on SNS will not harm me & - & - & - & - & $\mathbf{o}$ \\
\hline 30 & Cannot locate me in real life & - & - & - & - & $\mathbf{o}$ \\
\hline 35 & There can be threats of racism & - & - & - & - & $\mathbf{0}$ \\
\hline 36 & There can be threats of spam or spim & - & - & - & - & $\mathbf{0}$ \\
\hline 44 & Good usability of privacy settings & - & - & - & - & $\mathbf{0}$ \\
\hline 8 & Expand business and professional network & - & - & - & - & - \\
\hline 19 & Not expose much info on this SNS & - & - & - & - & - \\
\hline 23 & Trust protective service and function of SNS & - & - & - & - & - \\
\hline 26 & SNS has multiple privacy settings & - & - & - & - & - \\
\hline 27 & Share info about religion or political points of view & - & - & - & - & - \\
\hline 45 & Good usability of SNS & - & - & - & - & - \\
\hline 46 & Find more info about people at school or workplace & - & - & - & - & - \\
\hline
\end{tabular}

Table A2: List of distinguishing/ consensus items (statements) 
Copyright: (C) 2015 Dang-Pham, Pittayachawan \&Nkhoma. This is an open-access article distributed under the terms of the Creative Commons Attribution-NonCommercial 3.0

Australia License, which permits non-commercial use, distribution, and reproduction in any medium, provided the original author and AJ IS are credited.

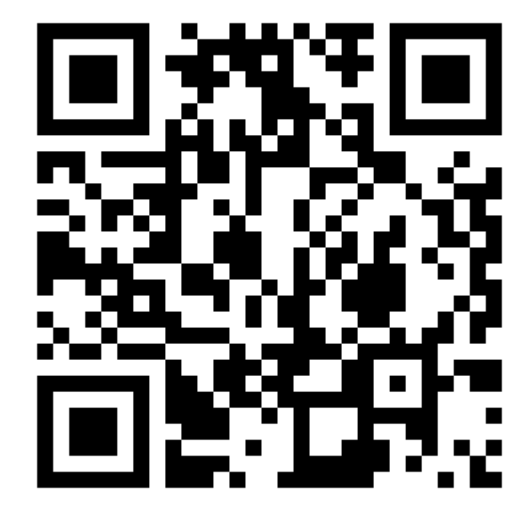

